chronic schizophrenia improving during pregnancy when estrogen levels are extremely high.

Methods: A double-blind, 28-day, placebo-controlled, adjunctive study was conducted comprising two groups of women of childbearing age. While one group of women received $100 \mathrm{mcg}$ transdermal estradiol, the other group received transdermal placebo. The differences in psychopathology between the two groups were subsequently compared. Hormone, psychopathology and cognitive assessments were performed routinely throughout the 4-week trial period.

Results: Using the Positive and Negative Syndrome Scale (PANSS) rating scale, it was noted that women receiving $100 \mathrm{mcg}$ estradiol improved significantly more in terms of their psychotic symptoms compared with women receiving placebo. Importantly, women who received estradiol improved with regard to positive, negative and general symptoms on the PANSS, in contrast to women on the placebo arm.

Conclusions: Estradiol appears to be a useful treatment for women with schizophrenia. We are furthering this exciting area of research by conducting a multisite 'proof-of-concept' study to determine whether estradiol can be used as an adjunctive treatment of psychotic symptoms in women with schizophrenia.

\section{Using theories of delusion formation to explain abnormal thinking in patients with body dysmorphic disorder}

\section{Labuschagne', D Castle², M Kyrios ${ }^{3}$, S RosselI'}

'Mental Health Research Institute of Victoria; ${ }^{2}$ St Vincent's Hospital, Melbourne; and ${ }^{3}$ Swinburne University, Melbourne, Australia

Background: Body dysmorphic disorder (BDD) is characterized by an overvalued or delusional belief of 'imagined ugliness'. According to the literature, delusional beliefs have been explained by four alternative theories, which include faulty perceptions, theory of mind deficits, reasoning abnormalities and corruption of semantic memory. The current study examined whether these potential explanations are relevant to delusion formation in BDD.

Method: Preliminary data from 10 BDD patients and 10 matched healthy controls were analyzed. The clinical assessment involved questionnaires measuring self-esteem, self-ambivalence, delusional thinking and creative experiences. The cognitive test battery included visual affect perception, semantic memory for somatic concepts, cognitive inhibition associated with somatic and nonsomatic words, and language fluency. Results: The results confirmed previous findings that patients with BDD are more delusional but additionally showed that delusional beliefs are exceedingly distressing and preoccupying for these patients. Similarly, on a semantic memory task, patients with BDD showed greater acceptance of unusual ideas especially with regard to somatic compared with neutral information. On the fluency task, patients with BDD showed impaired semantic fluency but intact phonological fluency. Furthermore, patients with BDD were impaired in recognizing angry facial expressions, with no deficits on identifying other emotions.

Conclusions: These results have indicated the influence of delusional thinking on cognitive processing in BDD. They have suggested that delusional beliefs may be explained in terms of impaired semantic memory and faulty perception of angry information; these deficits in turn may explain the specificity of preoccupations in BDD.

\section{One-year estimate of depot antipsychotic adherence and readmission in Victorian community mental health settings}

\section{T Lambert', A de Castella², A-n Ong ${ }^{3}$, J Kulkarni², B Sing $^{4}$}

'OPEN, The University of Melbourne and ORYGEN Research Centre; ${ }^{2}$ Alfred Psychiatric Research Centre, The Alfred and Monash University; ${ }^{3}$ Northwestern Mental Health; and ${ }^{4}$ The University of Melbourne, Australia

Background: This study aimed to 1) to establish the actual or 'found' depot adherence rate in typical community psychiatric settings, 2) to describe the sociodemographic associations with depot adherence and 3) to investigate the relationship between the degree of depot adherence and admission rate to hospital.

Methods: Patients treated with depot antipsychotics were sampled from CCT settings in two AMHSs in urban Melbourne. Depot adherence was defined as patients receiving their injection \pm 7 days from the due injection date. Sociodemographic data were acquired from relevant administrative databases.

Results: The study finds that there is a high mean adherence rate $(93 \%)$ and the rate of complete adherence is $54 \%$. Patients' adherence was not related to gender, being subject to a CTO, being of NESB, long durations of illness or time on depot treatment. Twenty-eight per cent were admitted in the study year and admission was significantly inversely proportional to depot adherence. The risks of readmission increase significantly when patients are less than $85 \%$ adherent, having a relative risk of readmission of 2.63 , and for those with less than $75 \%$ adherence, a relative risk of 4.32 $(P<0.01)$.

Conclusions: To our knowledge, this is the first study to report on the FDAR in community-treated patients. 
The finding that below $85 \%$ adherence, readmission is significantly more likely suggests that there may be a role for carefully and progressively monitoring depot adherence in community services. Reduction in relapses from enhanced adherence will have clinical, social and economic benefits.

\section{The metabolic syndrome in patients with a prolonged psychotic illness within a community setting: age and gender issues}

\author{
T Lambert ${ }^{1,2,3}$, C Pantelis ${ }^{4}$, N Freeman' \\ ${ }^{1}$ Office for Psychiatric Evaluation and Educational NewMedia (OPEN); ${ }^{20 R Y G E N ; ~}$ \\ ${ }^{3}$ The University of Melbourne ${ }^{4}$ Melbourne Neuropsychiatry Centre, \\ The University of Melbourne, Melbourne, Australia
}

Background: To measure the prevalence of the metabolic syndrome among patients with a prolonged psychotic illness being treated within a community setting and to examine gender- and age-stratified trends.

Methods: The study sample consisted of patients receiving treatment in the midwest or northwest mental health regions of Melbourne, Australia, between February 2003 and February 2004. Of the 206 patients aged 18 years and over who were approached, 106 consented to participate, yielding a response rate of $52.7 \%$. Participants were assessed for the presence of metabolic syndrome using the Adult Treatment Panel Guidelines-III).

Results: Prevalence of the metabolic syndrome among patients within this population was $39.4 \%$ (an alternate method of determining the denominator, indicates a rate of $\sim 50 \%$ ). Patients with the metabolic syndrome had a higher body mass index (31.73 vs. 28.82, $P<0.01)$ and increased triglyceride levels (3.49 vs. $1.70, P<0.01)$ compared with patients without this syndrome. Men with the metabolic syndrome had lower mean high-density lipoprotein cholesterol levels $(0.95$ vs. $1.24, P<0.01)$ compared with men without this syndrome. In general, there were distinct gender patterns of abnormality in the metabolic components, and age stratification shows increased relative risks in the young as contrasted with the older patients.

Conclusions: The prevalence of the metabolic syndrome is high among people with a prolonged psychotic illness. Health professionals treating people within this population need to be monitoring their patients' physical health as well as looking after their mental well-being.

\section{Stability of antipsychotic prescribing: description and relationship to readmission}

\author{
T Lambert ${ }^{1,2}$, B Singh ${ }^{2}$ \\ 'OPEN/ORYGEN; and ${ }^{2}$ The University of Melbourne, Melbourne, Australia
}

Background: As showed by the CATIE study, antipsychotic prescribing/switching stability appears to be less than robust. Little is known of longitudinal stability in other treatment cultures. This paper presents Australian data to outline trends in routine clinical practice of CCT-treated patients.

Methods: A cohort abstracted from our large database is described. Prescribing stability and readmission were examined in patients with schizophrenia treated with antipsychotic monotherapy at $\mathrm{T} 1$ and $\mathrm{T} 2$ (18 months panel data). Of the 817 patients, 302 were on monotherapy at both times.

Results: A matrix of prescribing/switching stability indicating the rate of persistence on one medicine and, if switched, to what antipsychotic will be presented. Eighteen-month monotherapy persistence rates were as follows: clozapine $81.3 \%$, olanzapine $71.0 \%$, FGA depots $65.3 \%$, risperidone $51.4 \%$ and FGA orals $34.0 \%$. Numerically, the largest switching traffic occurred from depot to olanzapine and vice versa. Readmission, only $13.4 \%$ were readmitted. Being on a depot at T1 was 2.33 times more likely than being on an oral to result in admission in the study period $(P=0.004)$. If clozapine is excluded, there is no difference between depots and SGAs (RR 1.62, $P>0.05$ ). Overall readmission was 2.15 times more likely to occur in those in whom antipsychotic switching occurs (direction of causality undetermined).

Conclusions: Stability is somewhat higher in Australia than reported for the United States. However, the general comparative trends in terms of the various antipsychotics are supported. Clozapine and olanzapine appear to have particularly stable use.

\section{Psychostimulant withdrawal: natural history and options for intervention}

\section{N Lee, A Harney, L Johns, A Pennay, P Kenny}

Turning Point Drug and Alcohol Centre, Melbourne, Australia

Background: Psychostimulant withdrawal is still not well understood. Much of the limited literature has been in the cocaine area and very little with methamphetamine. In particular, the natural history of withdrawal from psychostimulants is not well documented 